\title{
Bilateral Adie's Pupil following Laser Treatment of the Ischemic Peripheral Retina for Uveitis: A Case Report
}

\author{
Satoko Akahane Takao Hirano Sayuri Shu Toshinori Murata \\ Department of Ophthalmology, Shinshu University School of Medicine, Matsumoto, Japan
}

\section{Keywords}

Adie's pupil · Uveitis · Laser photocoagulation · Peripheral retinal nonperfusion

\begin{abstract}
Adie's pupil is a neurological condition of unknown origin with unusual, asymmetric presentation known as anisocoria with the enlarged pupil failing to react to light. It is believed that this pupillary abnormality results from damage to the ciliary ganglion or postganglionic short ciliary nerves. Affected individuals (usually female) may be symptomatic with photophobia or difficulty reading in the diseased eye. Although most Adie's pupil cases are idiopathic, previous studies have associated photocoagulation and uveitis with symptom onset. To the best of our knowledge, there have been no reports of specific means of preventing Adie's pupil. We describe a patient who experienced varying severities of Adie's pupil after separate laser treatments of the ischemic peripheral retina for uveitis. Fluorescein angiography revealed peripheral retinal nonperfusion in the bilateral eyes of a 37-year-old Japanese female who had been suffering from posterior uveitis. To avoid proliferative changes, $360^{\circ}$ laser photocoagulation of the retinal nonperfusion region located in the far periphery was first delivered to the left eye over 2 sessions. Soon after treatment, the patient complained of acute photophobia and blurred vision in the treated eye. Ocular examination revealed left pupil dilation and poor light sensitivity, although the pupil was reactive to a close stimulus. The left pupil also displayed positive denervation sensitivity based on the dilute pilocarpine $(0.125 \%)$ test. Adie's pupil was diagnosed based on these observations. Three months later, similar, albeit milder, findings were observed in her right eye after $360^{\circ}$ peripheral laser photocoagulation that was more conservatively performed over 4 sessions. Four months after the first treatment, her subjective visual function had improved, and the pupil diameter had decreased to a normal size in both eyes without additional treatment. We encountered a patient whose severity of
\end{abstract}


Adie's pupil was apparently reduced by more conservative laser photocoagulation of the ischemic peripheral retina.

\section{Introduction}

Patients with Adie's pupil often discover that they have a unilateral partially dilated pupil. Affected individuals are usually otherwise healthy and more commonly female. Patients often complain of blurred vision of nearby targets. Most patients do not require any treatment [1]. The condition is defined as an isolated iris sphincter and ciliary muscle dysfunction resulting from damage to the ciliary ganglion or postganglionic short ciliary nerves in the orbit that is followed by aberrant reinnervation. Although most Adie's pupil cases are idiopathic, previous studies have described photocoagulation and uveitis cause Adie's pupil [2-6]. To the best of our knowledge, there have been no reports of specific means of preventing Adie's pupil. We describe the clinical findings of a patient with bilateral Adie's pupil manifesting after laser treatment of the ischemic peripheral retina in both eyes for uveitis.

\section{Case Report}

The patient was a 37-year-old Japanese female who had been suffering from posterior uveitis in both eyes. She was referred to our department. Her best-corrected visual acuity (BCVA) was 20/13 OD and OS, respectively. Intraocular pressure was $10 \mathrm{~mm} \mathrm{Hg} \mathrm{OD} \mathrm{and}$ $11 \mathrm{~mm} \mathrm{Hg}$ OS. The anterior chambers were deep and clear in both eyes. Fundus examination revealed a clear vitreous along with several retinal hemorrhages and exudates on the peripheral retina and post pole (Fig. 1a, b). HLA-B51 testing was positive. The patient had a single aphthous ulcer and vulvar ulcer at the time of initial uveitis onset, but no recurrence was observed thereafter. Therefore, the patient did not meet the international diagnostic criteria for Behçet's disease and was only suspected to have it. Fluorescein angiography (FA) demonstrated a small amount of leakage with peripheral retinal nonperfusion in both eyes (Fig. 1c, d). Although we suggested colchicine and immunosuppressive drugs to the patient, she refused these medications, since she was planning to conceive a child. To avoid proliferative changes, $360^{\circ}$ laser photocoagulation of the peripheral retinal nonperfusion region was first performed in the left eye over 2 sessions using MC-500 ViXi ${ }^{\circledR}$ (NIDEK Inc., Gamagori, Japan). At follow-up 1 week postoperatively, the patient complained of acute photophobia and blurred vision in the treated eye. Ocular examination revealed left pupil dilation and poor light sensitivity (Fig. 2a, b), although the pupil was reactive to a close stimulus. At 45 min after administration of $0.125 \%$ pilocarpine drops onto both conjunctivas, the left pupil had become constricted (Fig. 2d) while the right had not (Fig. 2c). Her deep tendon reflexes were normal. Based on these observations, a diagnosis of Adie's pupil was made, although we suspected that nerve damage caused by the photocoagulation procedure may have contributed to its pathogenesis. Three months later, $360^{\circ}$ laser photocoagulation of the peripheral retinal nonperfusion region was more conservatively performed over 4 sessions in the right eye using MC-500 ViXi ${ }^{\circledR}$. Afterward, milder Adie's pupil symptoms were witnessed in the treated eye. Ocular examination showed the right pupil to be irregularly shaped and slightly dilated. It was partially affected by $0.125 \%$ pilocarpine (Fig. 3a, b). Four months 


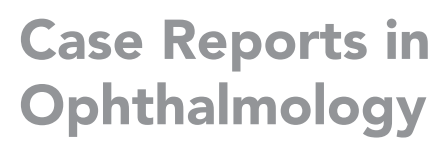

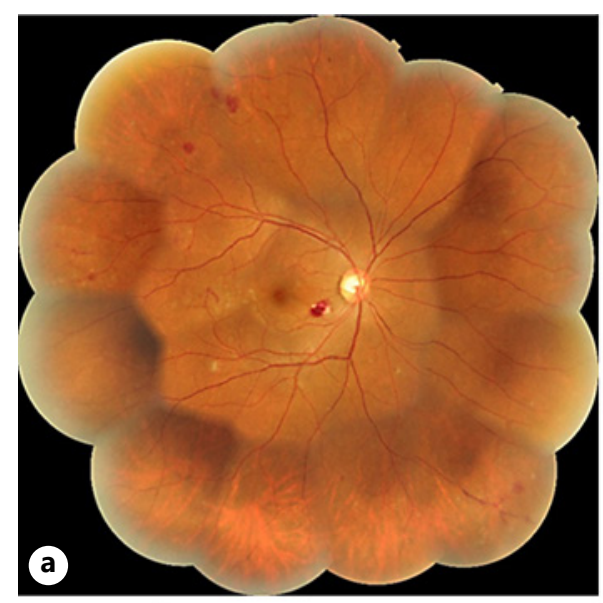
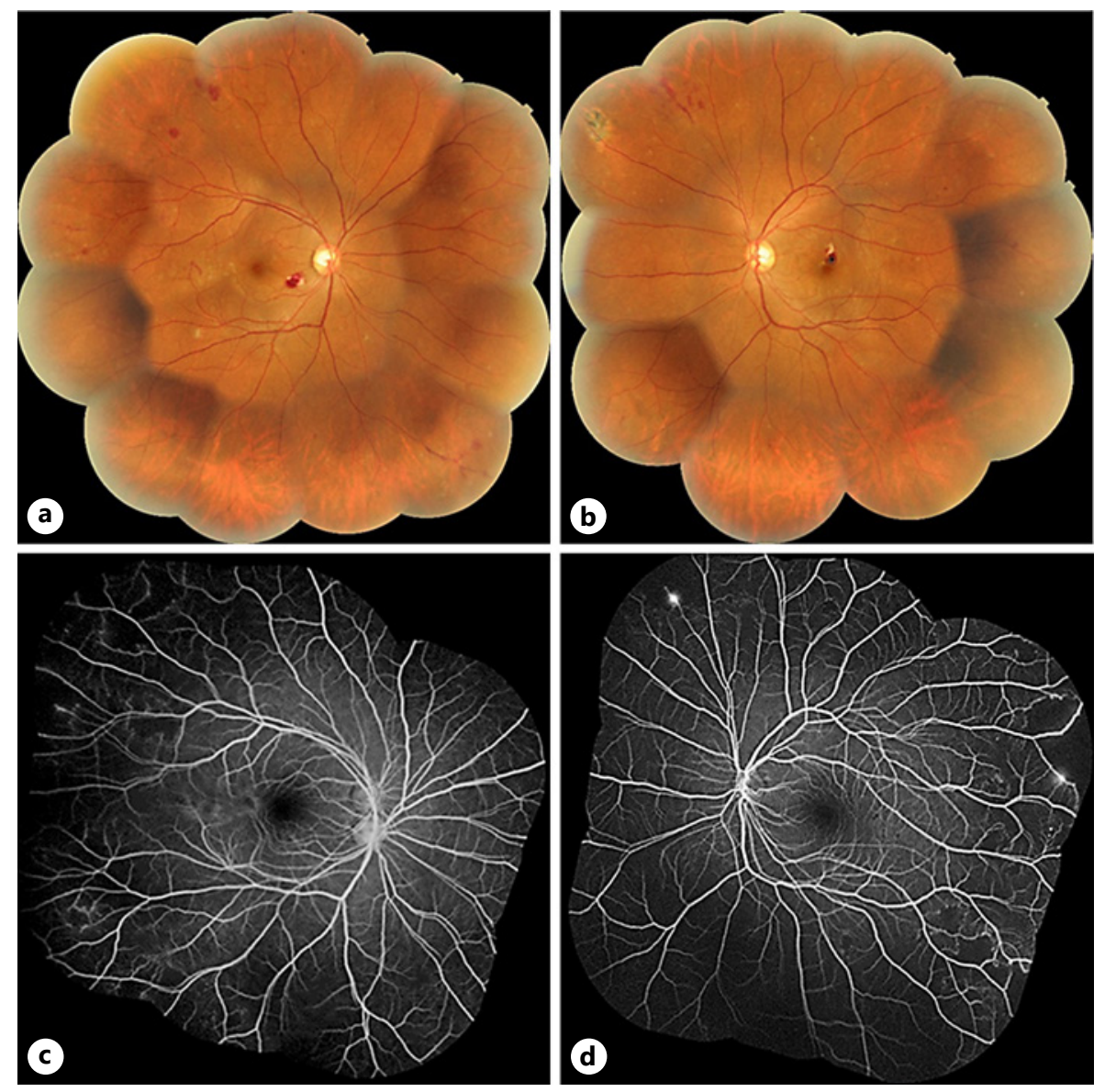

Fig. 1. Color fundus photo and FA before photocoagulation. Color fundus photo revealed a clear vitreous along with several retinal hemorrhages and exudates in both eyes $(\mathbf{a}, \mathbf{b})$. FA showed a small amount of leakage with peripheral retinal nonperfusion in both eyes $(\mathbf{c}, \mathbf{d})$.

after the first treatment, her subjective visual function had improved, and the bilateral pupil diameters had decreased to a normal size without additional treatment. Her BCVA was 20/13 OD and OS, respectively, and pupil diameter was $3.5 \mathrm{~mm}$ OD and OS, respectively (Fig. 4a, b). Fundus photography confirmed successful laser scarring of the peripheral retina of both eyes (Fig. 4c, d).

\section{Discussion}

We encountered a patient who experienced ipsilateral Adie's pupil after laser treatments of the ischemic peripheral retina for uveitis. The hallmark clinical signs of Adie's pupil are a large pupil that does not react or reacts poorly to light, a more definite response to nearby targets (i.e., light-near dissociation), slow tonic redilation of the pupil from near to far targets due to sphincter muscle denervation supersensitivity, and sensitivity to dilute pilocarpine $(0.125 \%)$. Pharmacologic testing typically confirms the diagnosis of Adie's pupil. The causes of this disease fall into 3 major groups: Adie syndrome, local ocular processes, and autonomic dysfunction. Adie syndrome is a symptom complex consisting of Adie's pupil and absent deep tendon reflexes [7]. Meanwhile, local ocular processes affect the ciliary ganglion or short 


\section{Case Reports in Ophthalmology}
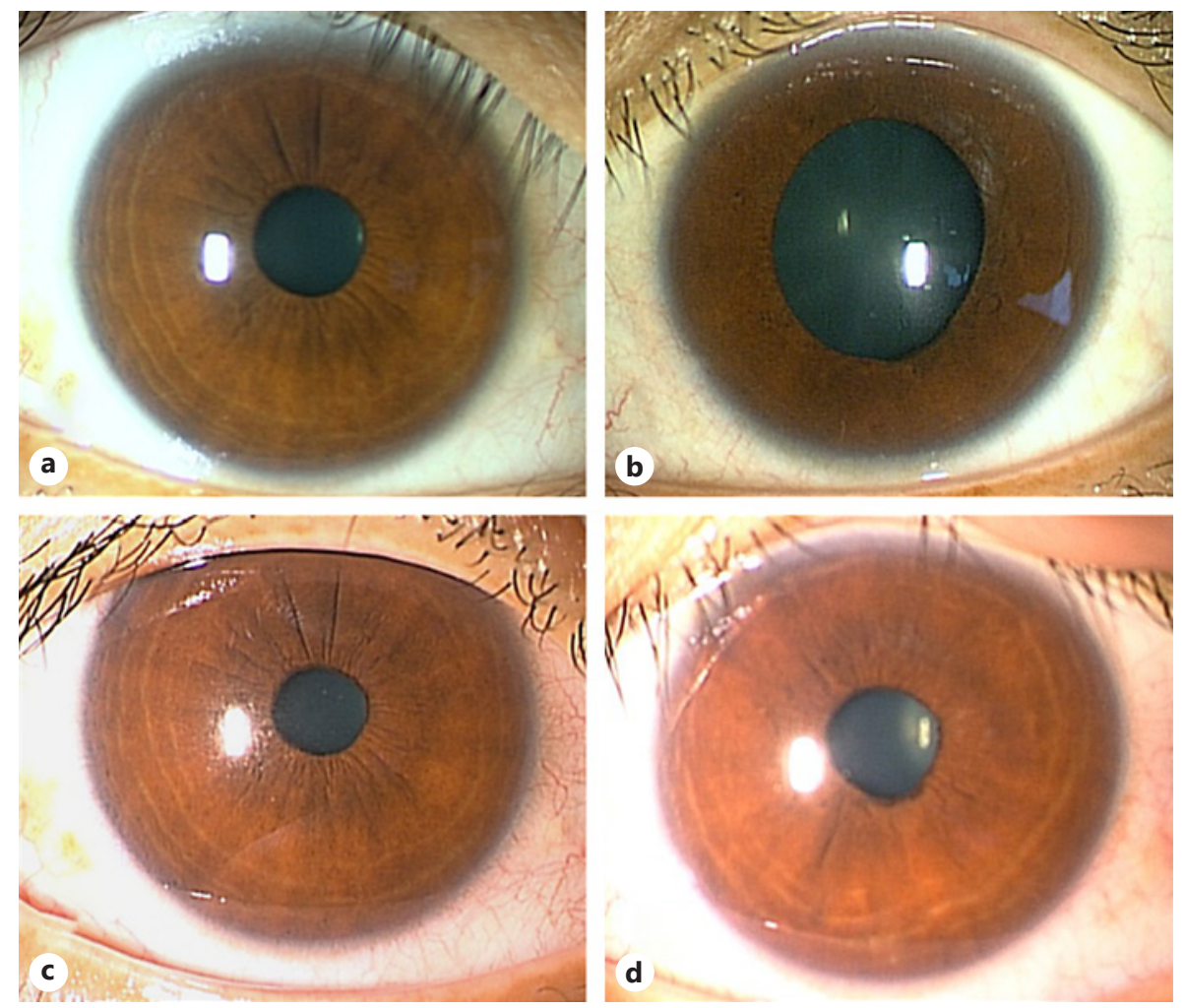

Fig. 2. Adie's pupil in the left eye. The left pupil $(6.5 \mathrm{~mm})$ was in midposition and was larger than the right pupil (3.5 mm) (a, b). At $45 \mathrm{~min}$ after administration of $0.125 \%$ pilocarpine drops onto both conjunctivas, the left pupil (d) had become constricted, while the right (c) had not.
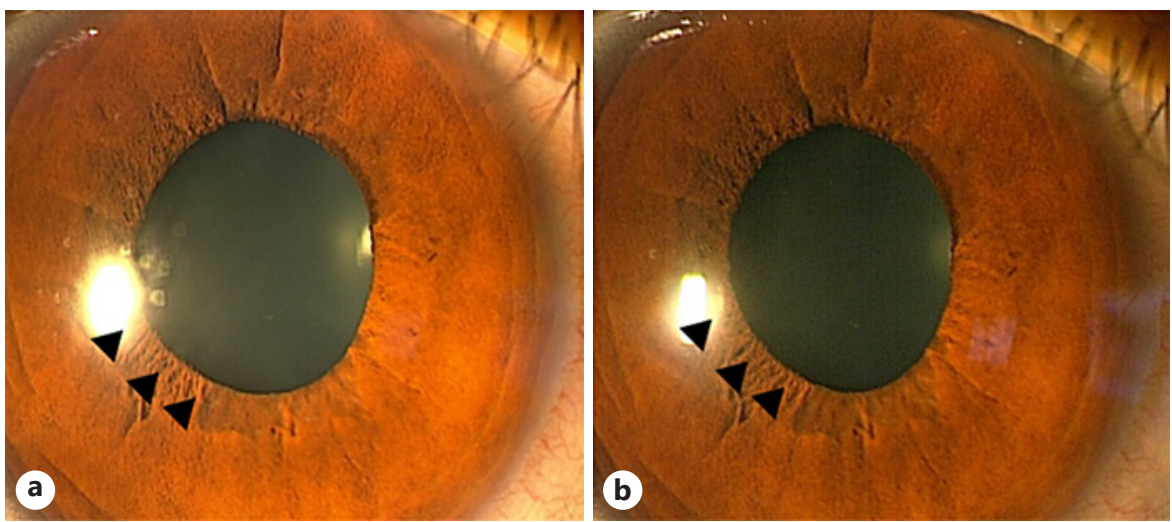

Fig. 3. Adie's pupil in the right eye. The right pupil was irregularly shaped and mildly dilated (a). A dilute pilocarpine $(0.125 \%)$ test partially (arrowhead) affected the right pupil (b).

ciliary nerves and include eye or orbital trauma, sarcoidosis [4], viral illnesses, ischemia [8], and laser treatment for proliferative diabetic retinopathy [2]. Regarding autonomic dysfunction, Adie's pupil may uncommonly occur in association with conditions such as neurosyphilis, advanced diabetes mellitus, and dysautonomias. It is also well known that Adie's pupil is a rare complication of panretinal photocoagulation for diabetic retinopathy; thermal injury to the parasympathetic motor nerves running in the short ciliary nerves anteriorly through the 


\section{Case Reports in Ophthalmology}
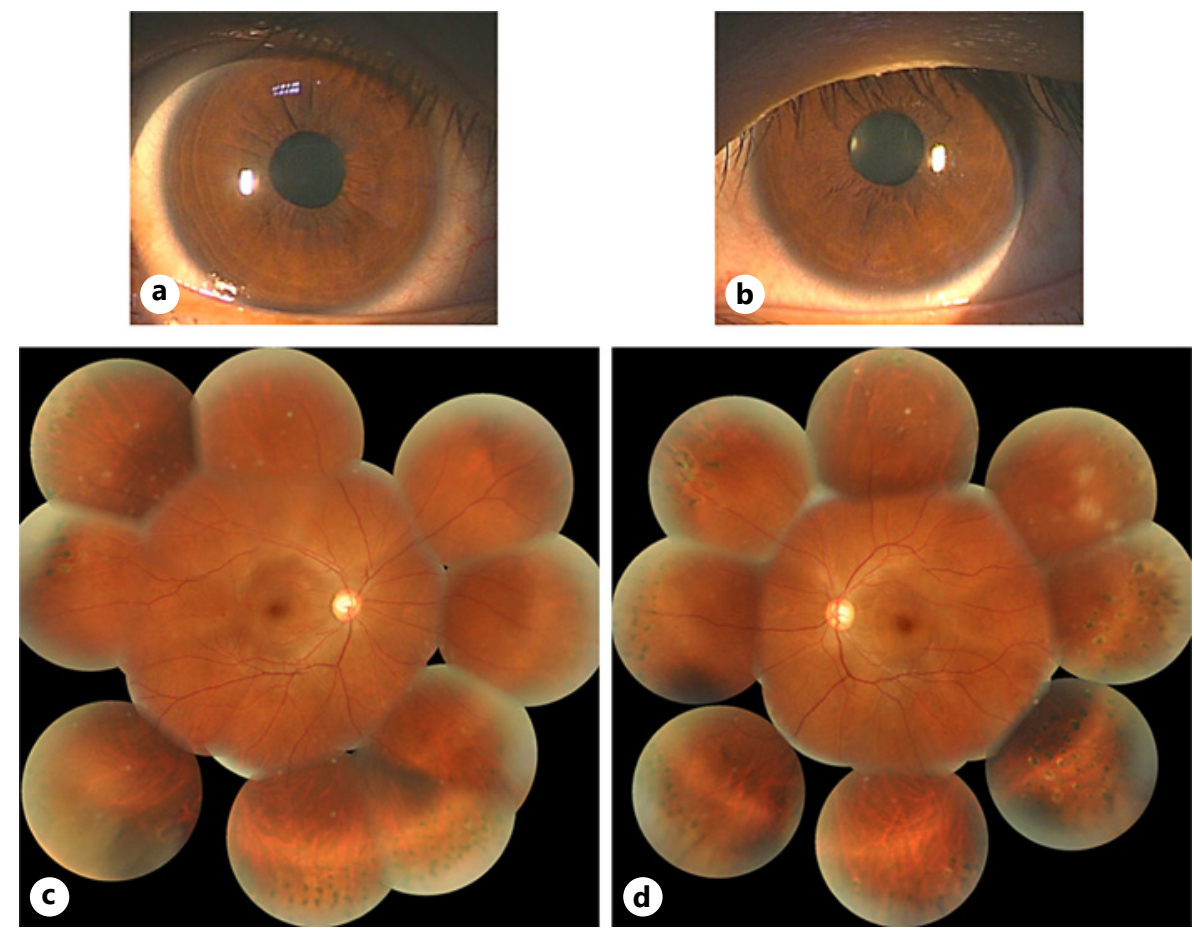

Fig. 4. Anterior segment and color fundus photo after photocoagulation. The pupil diameters had decreased to normal size $(3.5 \mathrm{~mm})$ in both eyes without additional treatment $(\mathbf{a}, \mathbf{b})$. A fundus photo confirmed laser scarring of the peripheral retinas in both eyes $(\mathbf{c}, \mathbf{d})$.

choroid and suprachoroidal space could explain this complication [3]. Several reports have described Adie's pupil in patients with uveitis (Harada disease [5] and iridocyclitis [6]) as well as in whom uveitis-associated inflammation affected the ciliary ganglion or short ciliary nerves. In the present case, laser photocoagulation of the periphery of the retinas may have led to reversible damage to the underlying long posterior ciliary nerve. Our patient experienced ipsilateral Adie's pupil after both photocoagulation procedures despite performing therapy more gradually in the right eye (703 shots over 4 sessions) than in the left (754 shots over 2 sessions). However, Adie's pupil was far less severe in the right eye. Accordingly, dividing laser photocoagulation therapy for uveitis into several smaller sessions may reduce inflammation and damage to the surrounding nerves and ensuring Adie's pupil.

\section{Conclusion}

We encountered a patient who experienced ipsilateral Adie's pupil after laser treatment of the ischemic peripheral retina for uveitis. Laser photocoagulation of eyes with uveitis can cause local inflammation around the ciliary ganglion or postganglionic short ciliary nerve systems, thereby predisposing the patient to Adie's pupil.

\section{Acknowledgment}

The authors thank the patient who kindly agreed to participate in this report.

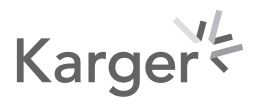




\section{Statement of Ethics}

The study was approved by the Ethics Committee of the Shinshu University School of Medicine (Approval No. 2044) and adhered to the tenets set forth in the Declaration of Helsinki. Written informed consent was obtained from the patient for publication of this case report and accompanying images.

\section{Conflict of Interest Statement}

The authors do not have any conflicts of interest to declare.

\section{Funding Sources}

This manuscript did not receive any funding.

\section{Author Contributions}

S.A. drafted the manuscript, collected the data, and reviewed the literature. T.H. was involved in the design of the study, interpretation of the data, drafting of the manuscript, and review of the literature. S.S. and T.M. participated in the design of the study, collection of the data, interpretation of the data, and review of the literature. T.M. drafted the manuscript, interpreted the data, and critically reviewed the manuscript. All authors have read and approved the final manuscript.

\section{Data Availability Statement}

All data generated or analysed during this study are included in this article..

\section{References}

1 Bourgon P, Pilley FJ, Thompson HS. Cholinergic supersensitivity of the iris sphincter in Adie's tonic pupil. Am J Ophthalmol. 1978 Mar;85(3):373-7.

2 Rogell GD. Internal ophthalmoplegia after argon laser panretinal photocoagulation. Arch Ophthalmol. 1979; 97(5):904-5.

3 Patel JI, Jenkins L, Benjamin L, Webber S. Dilated pupils and loss of accommodation following diode panretinal photocoagulation with sub-tenon local anaesthetic in four cases. Eye. 2002 Sep;16(5):628-32.

4 Bowie EM, Givre SJ. Tonic pupil and sarcoidosis. Am J Ophthalmol. 2003 Mar;135(3):417-9.

5 Kim JS, Yun CH, Moon CS. Bilateral tonic (Adie's) pupils in Vogt-Koyanagi-Harada syndrome. J Neuroophthalmol. 2001 Sep;21(3):205-6.

6 Puri P, Verma D. Bilateral glaucomatocyclitic crisis in a patient with Holmes Adie syndrome. J Postgrad Med.1998 Jul-Sep;44(3):76-7.

7 Martinelli P. Holmes-Adie syndrome. Lancet. 2000 Nov 18;356(9243):1760-1.

8 Bennett JL, Pelak VA, Mourelatos Z, Bird S, Galetta SL. Acute sensorimotor polyneuropathy with tonic pupils and an abduction deficit: an unusual presentation of polyarteritis nodosa. Surv Ophthalmol. 1999;43(4):341-4.

\section{Karger' ${ }^{\prime \prime}$}

